\title{
ClickMath: Editor matemático para o ambiente virtual de aprendizagem Moodle
}

\author{
Rodrigo Nascentes da Silva - ULBRA - roudrigon@gmail.com \\ Maurício Rosa - ULBRA - mauriciomatematica@gmail.com
}

\section{Resumo}

Representar uma expressão matemática em documentos online pode não ser uma tarefa simples, mesmo com os avanços da tecnologia nos dias atuais, principalmente em função do uso de símbolos e fórmulas específicos. Este artigo apresenta o ClickMath, um protótipo de editor matemático para o ambiente virtual de aprendizagem Moodle. Este protótipo utiliza os benefícios da linguagem MathML para uma comunicação matemática efetiva e facilitada pela Internet. Assim, o objetivo principal da criação desse editor é possibilitar a comunicação matemática online efetiva, de forma rápida e com boa usabilidade.

Palavras-chave: Educação matemática, educação a distância, mathml, ambientes virtuais de aprendizagem.

\section{Abstract}

\section{ClickMath: Mathematical editor for virtual learning environment}

To represent a mathematical expression on the web documents which requires its own notation and symbology might not be a simple task even with the technology's advances on the current days. So, this paper describes the benefits of the MathML for the effective mathematics communication through the Internet, as well as, proposes the implementation of a mathematical editor for virtual learning environment - Moodle. The editor's objective is to raise a fast, easy and effective mathematics communication.

Key-words: Mathematics education, distance education, mathml, virtual learning environments.

\section{I - Introdução}

$\mathrm{O}$ advento da Internet proporcionou que cursos, escolas e universidades obtivessem a abertura de novos espaços para desenvolver Educação a Distância (EaD), objetivando o ensino e a aprendizagem, tanto como apoio ao ensino presencial ou em modelos que funcionam completamente a distância, baseadas no uso de plataformas de comunicação à distância.

Dentre os diversos ambientes virtuais de aprendizagem, destaca-se o Moodle (do inglês, Modular Object-Oriented Dynamic Learning Environment), desenvolvido em 2002 por Martin Dougiamas com o objetivo de possibilitar uma educação baseada na Internet. É um programa construído sob os preceitos do Software Livre, ou seja, possui direitos autorais, mas pode ser copiado e alterado de acordo com os seus termos de uso e é desenvolvido por uma comunidade virtual formada por programadores, desenvolvedores, professores, usuários e parceiros dos mais diversos países (Moodle, 2009).

O Moodle é um programa que pode ser usado tanto para aulas totalmente online quanto como complemento à aprendizagem em sala de aula. Essa ferramenta é constituída por vários módulos para a construção de um ambiente virtual, tais como administração do site, administração do curso, administração dos usuários, módulo chat, módulo avaliação de curso, módulo diário, módulo glossário, módulo tarefas, entre outros. O Moodle tem a facilidade de ser modificado de acordo com as necessidades do 
administrador. Além disso, podem-se criar extensões adicionais para suprir uma determinada carência em um de seus módulos.

As inúmeras vantagens do uso da Internet como uma forma de comunicação e aprendizagem colaborativa ainda não podem ser observadas na sua plenitude no contexto do ensino da Matemática. Segundo Smith e Ferguson (2005), em pesquisa realizada com instrutores de cursos $\mathrm{EaD}$ do SUNY Learning Network, uma organização sem fins lucrativos que desenvolve mais de 3 mil cursos EaD para mais de 40 cursos de graduação da State University of New York, revela uma taxa de desistência de 31\% para cursos $\mathrm{EaD}$ relacionados à Matemática, enquanto para cursos não relacionados à Matemática a taxa de desistência é de 18\%. Ainda segundo Smith e Ferguson (2005), a alta taxa de desistência de cursos $\mathrm{EaD}$ relacionados à Matemática, em comparação com outros cursos $\mathrm{EaD}$ não relacionados a ela, deve-se, principalmente, às dificuldades ocasionadas pela falta de suporte adequado para o uso da notação matemática em ambientes virtuais de aprendizagem, como é o caso do Moodle, que possui suas áreas de entrada de texto, tais como recursos, postagens nos fóruns, criação de tarefas, questionários, entre outros, editadas usando um simples editor HTML WYSIWYG (do inglês, What You See Is What You Get - "O que você vê é o que você tem") incorporado (Moodle, 2009) . Esse editor, em seu formato original, não permite a inserção de notação matemática (Bazon, 2004).

\section{II - Comunicação Matemática na Internet}

Ainda hoje, a maioria das informações disponíveis na rede mundial de computadores é organizada em texto simples, sem a utilização de códigos especiais, devido a limitações dos primeiros dispositivos eletrônicos e computacionais. Isso acarretou uma dificuldade na representação de símbolos e notações matemáticas, muitas vezes compostas por fórmulas e caracteres especiais (Miner, 2005).

Até os dias de hoje, a maioria dos documentos com notação matemática é formada por textos com imagens e figuras de fórmulas e símbolos, cuja utilização tem, conforme Miner (2005), inúmeros inconvenientes:

- Podem apresentar baixa qualidade de exibição;

- Podem exigir ferramentas adicionais de manipulação de imagens, cujo conhecimento não é completamente difundido entre os professores;

- Não podem ser editados com os recursos disponíveis na formatação de fonte e parágrafo dos editores tradicionais; e

- Tornam o documento mais lento para carregar.

Ainda segundo Miner (2005), o problema de uma comunicação eletrônica efetiva de documentos contendo notação matemática é conhecido muito antes do surgimento da Internet. A necessidade desse tipo de comunicação fez com que fossem desenvolvidos inúmeros programas computacionais especializados no suporte de matemática. Entre eles, destaca-se o TeX, desenvolvido na década de 1970 por Donald E. Knuth, para a criação de livros e outras publicações como artigos e teses com excelente qualidade gráfica e muito eficaz na elaboração de documentos que contenham fórmulas matemáticas. Destaca-se, também, a ferramenta Maple, desenvolvida pelo Grupo de Computação Simbólica na Universidade de Waterloo, no Canadá, em 1981.

O Maple é um programa de álgebra computacional constituído de um ambiente informático para a criação e cálculo de expressões algébricas e simbólicas. Tanto o TeX 
quanto o Maple, por suas características avançadas no uso de representação matemática, ainda são, atualmente, muito utilizados em trabalhos na comunidade acadêmica, dentre os quais se destacam:

- "Utilização do Software Maple no Ensino-Aprendizagem de Cálculo" (Mariani, 2005);

- "Investigando alguns Desafios da Incorporação do Software Maple em Cursos Regulares do Ciclo Superior Inicial: Pré-Requisitos Algébricos e Avaliação" (Palis, 2007); e

- "O Estudo de Equações Polares Utilizando o Software Maple” (Kaiber; Renz, 2007).

Segundo Miner (1999), embora os diversos programas especializados em matemática, desenvolvidos há 20 ou 30 anos e ainda utilizados, contribuam para a comunicação eletrônica de conteúdo matemático, eles ainda apresentam uma série de restrições para uma comunicação efetiva usando a Internet. Geralmente, esses programas não se comunicam com outros, como é o caso do TeX e do Microsoft Word.

Além disso, o resultado da expressão gerada por esses programas continua sendo uma imagem gráfica. Também, muitos desses programas requerem licenças comerciais para o seu uso, como é o caso do Maple, que, além disso, exige uma configuração específica de hardware, sistema operacional ou plataforma. Essas restrições vão na contramão da regra de ouro da acessibilidade: "Qualquer pessoa, de qualquer lugar, desconsiderando plataforma, tecnologia, experiência ou capacidade, deve acessar o conteúdo principal" (Collison, 2008).

Também segundo Miner (1999), o World Wide Web Consortium, W3C, um consórcio de empresas de tecnologia que desenvolve padrões para a criação e a interpretação dos conteúdos para a Internet, motivado pela dificuldade de comunicação de conteúdo matemático começou, em 1994, uma série de estudos sobre como incluir notação matemática nos documentos da Internet, e em 1998 criou-se uma recomendação para a efetiva comunicação matemática na Internet, o MathML (do inglês, Mathematical Markup Language - Linguagem de Formatação Matemática).

O MathML é uma aplicação do XML, segundo a sua especificação editada por Ion e Miner (1999). O XML (do inglês, eXtensible Markup Language) é uma linguagem para a geração de formatação para necessidades especiais capaz de descrever diversos tipos de dados, e seu propósito principal é facilitar o compartilhamento de informações por meio da Internet (W3C, 2009). Ainda segundo sua especificação, o MathML é um tipo especial de dados para a representação de notação matemática tradicional, como símbolos, fórmulas, gráficos e matrizes nos diversos documentos da Internet. Assim, o MathML foi desenvolvido com os seguintes propósitos:

- Codificar material matemático para a educação e a comunicação científica;

- Codificar expressões matemáticas sem a perda do significado;

- Transmitir informação entre aplicações;

- Suportar eficientemente a leitura e navegação de longas expressões;

- Promover a extensibilidade; e,

- Ser legível para o ser humano e simples para o processamento de 
software.

Segundo Miner (2009), a expressão $(x+2)^{3}$ é codificada para apresentação em MathML como mostra a Figura 1.

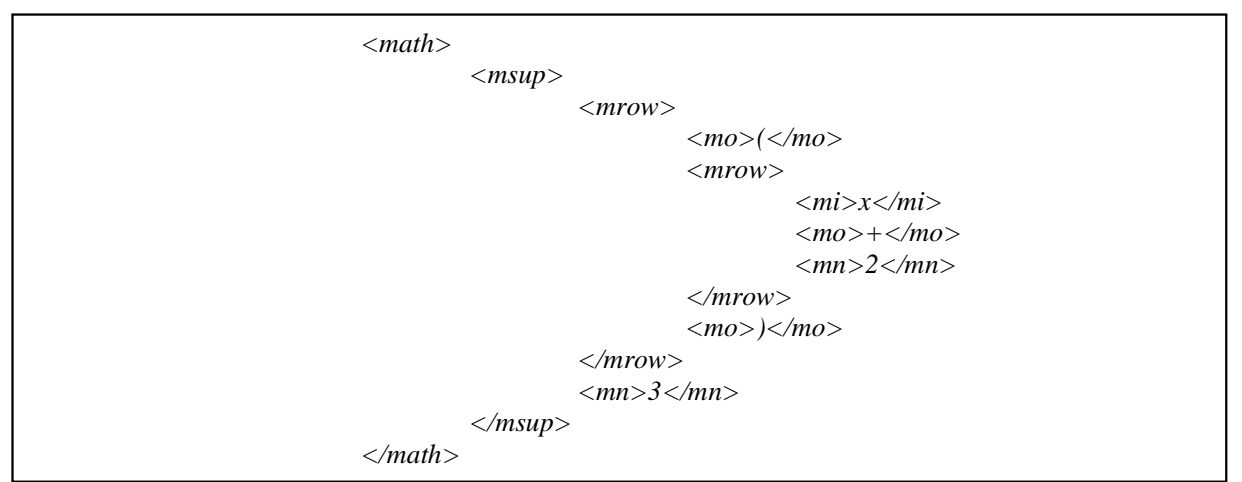

Figura 1 - Expressão $(x+2)^{3}$ em código de apresentação em MathML

\section{III - O Editor Matemático ClickMath}

Com o propósito de encontrar novas maneiras de possibilitar uma comunicação matemática efetiva para EaD e assim facilitar o processamento automático, busca, indexação e, principalmente, o reúso em outras aplicações matemáticas com o uso do MathML, foi projetado e desenvolvido o ClickMath, um protótipo de editor matemático online e integrado ao Moodle.

Para utilizar o ClickMath é necessário, primeiramente, o uso de um programa navegador de Internet que suporte MathML, como Mozilla Firefox ou o Microsoft Internet Explorer (Miner, 2009). O ClickMath, depois de instalado, encontra-se disponível junto à maioria das entradas de texto do Moodle. Assim, basta clicar no ícone com o símbolo somatório, como mostra a Figura 2, para se iniciar sua utilização, conforme a Figura 3.

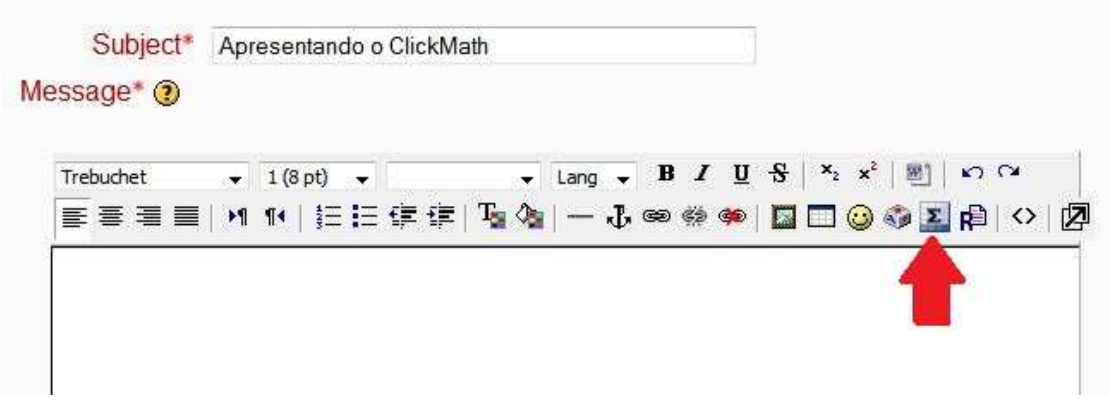

Figura 2 - Botão de atalho para acesso do ClickMath.

\section{[AS1] Comentário: Pela} ABNT isso agora deve vir em cima da figura propriamente dita. Ok? Mas confira na formatação da revista 


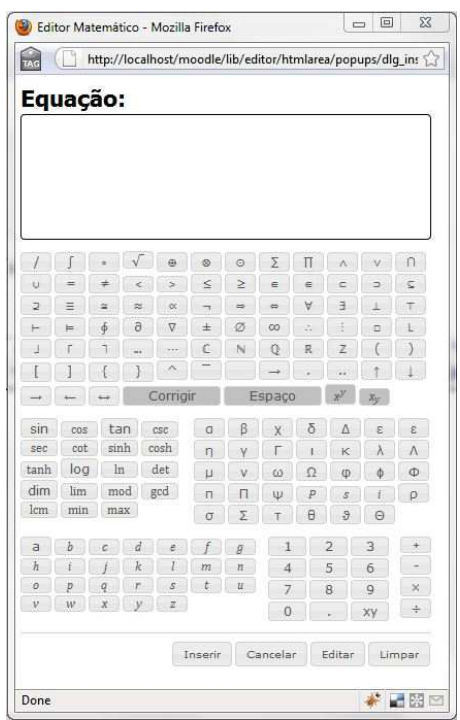

Figura 3 - O ClickMath.

Ao clicar nos botões do teclado virtual, a expressão matemática é automaticamente mostrada na área de texto superior. O processo para a montagem da expressão matemática $\int_{0}^{2} \sqrt{x^{3}+1} d x$ que será mostrada na Figura 4 encontra-se detalhado a seguir:

1. Primeiramente, o usuário clica no símbolo de integral $s$, e em seguida, para definir os limites de integração, clica no símbolo de subscrito $x_{y}$ e 0 para estabelecer o limite inferior e no símbolo de sobrescrito $x^{y}$ e 2, para o limite superior;

2. O usuário clica no símbolo de radiciação $\sqrt{ }$ e, logo após, $x$ usa novamente o símbolo de sobrescrito ${ }^{y}$ para elevar ao cubo;

3. O usuário clica em,+ 1 e em $\stackrel{+}{\perp}$ para fechar parênteses e encerra a expressão sub-raiz;

4. Para completar a equação, o usuário clica em $+\frac{d}{x}$; e

5. Para finalizar a edição, o usuário clica no botão Inserir e a equação será inserida na área de texto do Moodle, conforme pode ser observado na Figura 4.

Conforme se pode observar, a equação $\int_{0}^{2} \sqrt{x^{3}+1} d x$ descrita na Figura 3 pouco se parece com aquela mostrada na Figura 5. Isso acontece porque, na equação da Figura 4, ainda se utiliza de código ASCII ${ }^{1}$, sendo que o resultado final somente será observado após o salvamento do texto, como pode ser observado na Figura 5. 


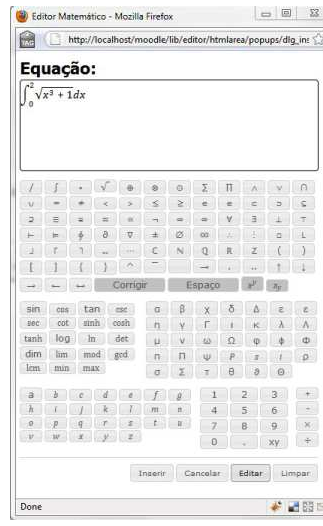

Figura 4 - Equação montada no ClickMath.

$$
\text { Subject* Re:Testando Forum }
$$

Message* (2)

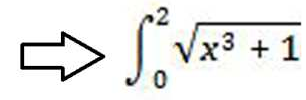

Trebuchet Dt Do

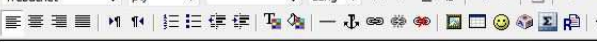
int_o 022 sart $\left(x^{\wedge} 3+1\right) d x^{\prime}$

Figura 5 - Equação inserida pelo ClickMath.

Figura 6 - Resultado final da equação escrita com o ClickMath.

No ClickMath, ainda é possível editar a equação em código ASCII, clicando no botão Editar, por meio do emprego de uma caixa de diálogo, como mostra a Figura 7.



Figura 7 - Caixa de edição da equação em código ASCII.

\section{IV - Análise do Editor Matemático ClickMath}

Um dos principais requisitos considerados para o desenvolvimento do ClickMath foi a utilização da linguagem MathML, versão 2.0, para a representação 
científica on-line em conformidade com os atuais padrões Web (W3C, 2009) e assim garantir a regra de ouro da usabilidade (Collison, 2008). Segundo Collison (2008), a utilização dos padrões Web traz muitos benefícios, já que reduz o tamanho das páginas, o que torna os downloads mais rápidos e, por sua vez, possibilita consumir menos banda. Os padrões Web também aumentam a compatibilidade com agentes de usuário, tais como navegadores, telefones celulares e softwares de ajuda, tornando os sites mais acessíveis.

Durante o desenvolvimento do editor, pensou-se numa forma de interação simples e intuitiva com a qual o usuário não tivesse a necessidade de conhecer MathML. Pois, conforme Notare e Behar (2009), "[...] numa situação de EaD, o objetivo principal é a aprendizagem de conceitos de um determinado domínio de conhecimento, e não a aprendizagem de linguagens necessárias a comunicação". Assim, se iniciou uma busca por métodos de abstração da linguagem MathML, ou seja, converter código ASCII em MathML. Logo o princípio de funcionamento do ClickMath é converter código ASCII para a linguagem MathML dinamicamente. Sendo o editor uma aplicação Web, para realizar a conversão dinâmica de ASCII para MathML foi necessária a utilização de biblioteca Javascript ${ }^{2}$.

Dentre as poucas encontradas para realizar a conversão proposta, destacam-se a MathJax $^{3}$, criada em 2009, como sucessora do antigo projeto jsMath, e a biblioteca ASCIIMathML desenvolvida pelo Dr. Peter Jipsen, professor da Chapman University (Jipsen, 2007). Pela facilidade de uso e, também, por ser utilizada atualmente em outros editores matemáticos para o Moodle, como o MathWriting ${ }^{4}$ e o DragMath ${ }^{5}$, optou-se pelo uso da biblioteca ASCIIMathML para o desenvolvimento do ClickMath. Além disso, esta biblioteca integra a recomendação do W3C para implementação da linguagem MathML (W3C, 2009).

No ClickMath, a interação usuário-editor é realizada por meio de um teclado virtual, contendo os símbolos mais utilizados na comunicação das ciências exatas, o que permite a inserção de símbolos para a composição de fórmulas por meio de um simples clique no mouse. A Figura 8 mostra a primeira interface gráfica do editor.

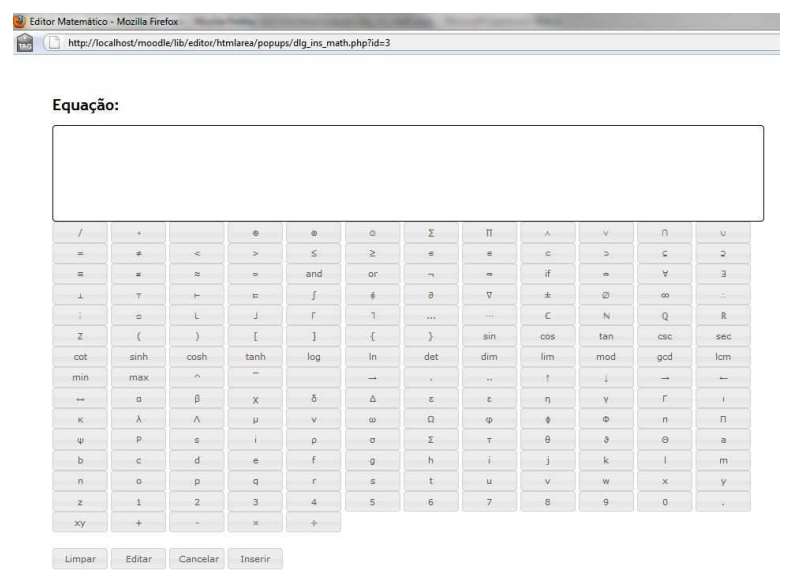

FIGURA 8 - Interface gráfica inicial do editor matemático ClickMath.

A programação de cada botão do teclado virtual é única e baseada na utilização de código HTML, ASCII e MathML, em conjunto com bibliotecas Javascript JQuery e JqueryUI, para facilitar os processos de configuração, montagem, layout e representação dos símbolos matemáticos, com mostra a Figura 9. 




Figura 9 - Código do botão com o símbolo de integração.

A linha um da Figura 9 mostra o conjunto de caracteres ASCII "int", os quais representam o símbolo matemático de integração, que ao clique no botão é anexado à área de texto acima do teclado virtual. As demais linhas mostram código HTML e MathML, que visto por meio de um programa navegador de Internet com suporte a MathML é assim mostrado na Figura 10.

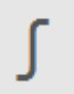

Figura 10 - Botão renderizado pelo programa navegador com suporte a MathML.

Para proporcionar dinamismo no momento em que o usuário insere um novo elemento, a biblioteca ASCIIMathML foi implementada para identificar o caractere ASCII, convertê-lo para MathML e assim, consequentemente, renderizá-lo pelo programa navegador. Além disso, tal implementação permite ao usuário uma completa manipulação da equação que deseja montar.

Embora o teclado fosse elaborado para uma interação rápida do usuário na comunicação matemática, tornando-a mais rápida por meio do clique do mouse, o layout inicial, mostrado na Figura 8, ainda não atendia a esse requisito. Uma vez que, em meio a inúmeros símbolos matemáticos, tornou-se demorado encontrar o símbolo desejado para montar a equação. Para suprir essa deficiência, procurou-se chegar a uma interface gráfica mais intuitiva, em que os botões fossem menores e agrupados por funcionalidade ou similaridade, como letras, números e símbolos, conforme pode ser observado na Figura 11.

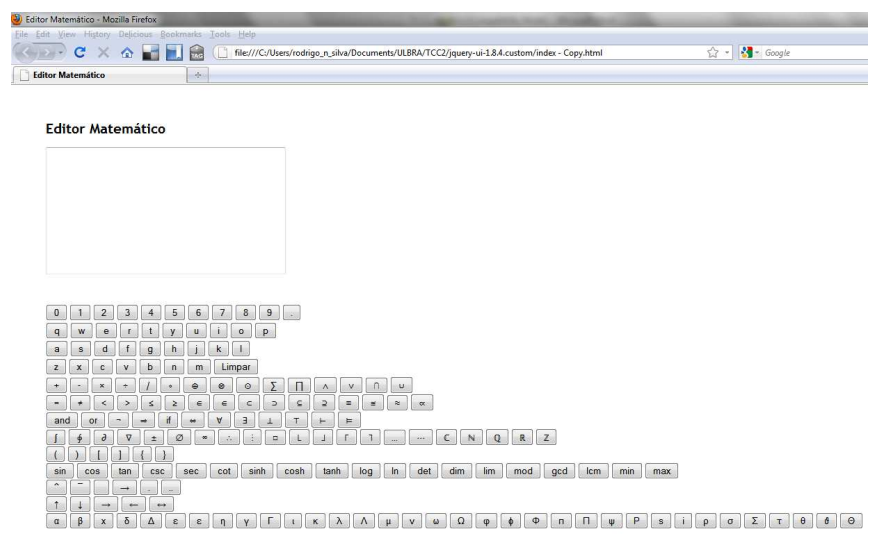

Figura 11 - Interface gráfica com os botões agrupados por funcionalidade ou similaridade.

A dificuldade em definir uma interface gráfica amigável e intuitiva levou à busca por padrões de design comuns a outros recursos tecnológicos. Logo, concluiu-se que a disposição dos botões de uma calculadora científica era o que melhor se aproximava do layout pretendido. Assim, foi desenvolvido um novo layout, como mostra a Figura 12. 

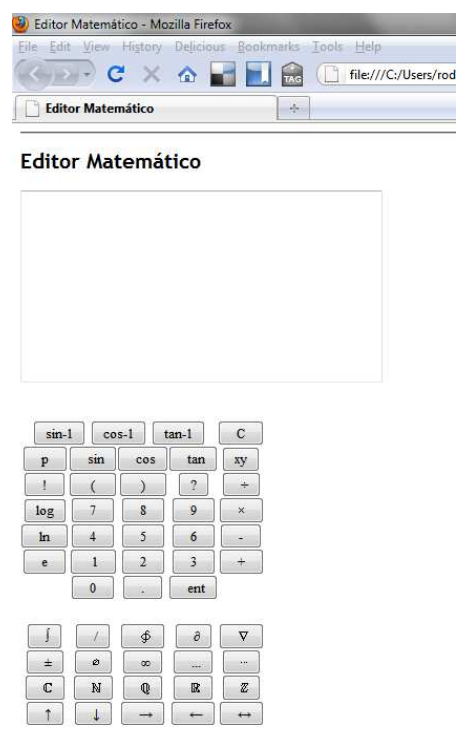

Figura 12 - Interface gráfica baseada em uma calculadora científica.

Após inúmeros aprimoramentos, a Figura 13 mostra o ClickMath em sua interface gráfica final.

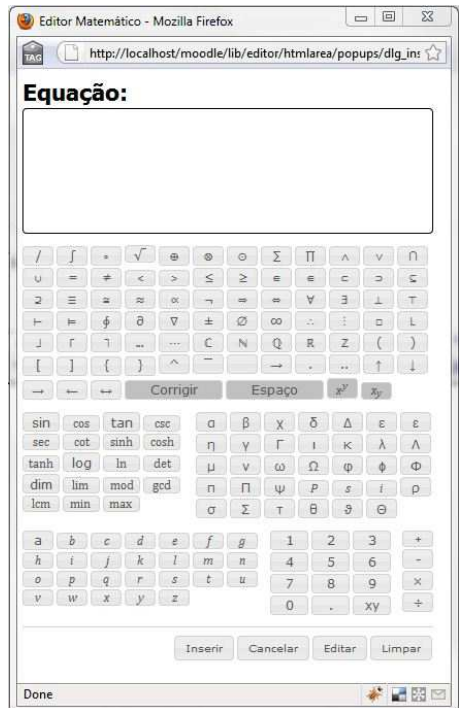

Figura 13 - Interface gráfica final e já integrada ao Moodle.

Apesar da dificuldade na definição da interface gráfica, o maior desafio encontrado durante o desenvolvimento do ClickMath foi a integração com o sistema Moodle. Apesar de o Moodle ser amplamente utilizado como ambiente virtual de aprendizagem por inúmeras instituições de ensino ao longo de seus oito anos de existência, ainda não é possível encontrar uma especificação técnica consistente e clara, necessária para atender a uma demanda de desenvolvimento. A maioria dessas informações encontra-se em fóruns e grupos de discussões espalhados pela Internet.

Desenvolver um editor matemático para um ambiente virtual de aprendizagem, como o ClickMath, com o principal objetivo de facilitar uma comunicação matemática 
efetiva para EaD é uma tarefa complexa e requer constante pesquisa e desenvolvimento. Sua atual interface gráfica ainda carece de melhorias de usabilidade. Melhorias essas que incluem a pré-disposição de equações mais utilizadas, a implementação de um sistema de ajuda e a possibilidade de incluir e manipular gráficos e matrizes. Além disso, é necessária uma pesquisa de usabilidade com alunos e professores, para a coleta de opiniões durante o uso do ClickMath, visando, assim, identificar as limitações do uso do editor no dia a dia dos cursos.

Uma vez que o ClickMath é integrado ao Moodle, torna-se plenamente viável a implementação desse editor com outros recursos dessa plataforma ainda não atendidos e que necessitem de uma melhor comunicação matemática, como, por exemplo, o chat. Além disso, o desenvolvimento de uma funcionalidade para a resolução de cálculos algébricos seria uma interessante área de pesquisa a ser desenvolvida dentro desse editor.

Por fim, um importante trabalho a ser realizado - e já iniciado pelo autor - é a inclusão do ClickMath como recurso oficial do Moodle, assim com o DragMath. Para isso, o ClickMath ainda precisa atender a uma série de requisitos técnicos, como documentação e testes de segurança, exigidos pela comunidade Moodle.org (Moodle, 2009).

\section{V - Considerações Finais}

Por certo ponto de vista, o ClickMath é meramente um outro editor matemático para ambiente virtual de aprendizagem. No entanto, o ClickMath implementa os últimos recursos tecnológicos como linguagens, bibliotecas e padrões para o desenvolvimento Web. A Educação Matemática ainda precisa fazer mais uso desses recursos, e, dessa maneira, o ClickMath contribui para a utilização desses recursos, significativos do ponto de vista da aprendizagem na era Google. Hoje, novas ferramentas de EaD são desenvolvidas na tentativa de promover espaços cada vez mais interativos, colaborativos, que favoreçam a comunicação efetiva entre os participantes. Embora muitas dessas ferramentas ainda estejam em fases iniciais de desenvolvimento, o futuro nos mostra um lugar de destaque para a comunicação matemática na Educação. Será este o caminho para favorecer o ensino de Matemática?

\footnotetext{
${ }^{1}$ O código ASCII (do inglês, American Standard Code for Information Interchange - "Código Padrão Americano para o Intercâmbio de Informação") é uma codificação de caracteres de oito bits baseada no alfabeto inglês. Os códigos ASCII representam texto em computadores, equipamentos de comunicação, entre outros dispositivos que trabalham com texto (ANSI, 2007).

${ }^{2}$ Uma biblioteca Javascript é um conjunto de código reutilizável, escrito em linguagem Javascript, cuja função é abstrair do desenvolvedor a programação exigida para criação de recursos de interatividade em uma página da Internet (SILVA, 2009)

${ }^{3}$ Projeto MathJax: http://www.mathjax.org

${ }^{4}$ Editor matemático MathWriting: http://limc.ufrj.br/mathwriting/P\%C3\%A1gina_principal

${ }^{5}$ Editor matemático DragMath: http://www.dragmath.bham.ac.uk/index.html
}

\section{VI - Referências Bibliográficas}

AMERICAN NATIONAL STANDARDS INSTITUTE - ANSI. Information Systems - Coded Character Sets - 7-Bit American National Standard Code for Information Interchange (7-Bit ASCII). New York. 2007. 
BAZON, Mihai. HTMLArea-3.0 Documentation. 2004. Disponível em < http://www.fccn.pt/htmlarea/reference.html >. Acesso em 17 set. 2010.

COLLISON, Simon. Desenvolvendo CSS na Web: Do Iniciante ao Profissional. Rio de Janeiro: AltaBooks. 2008. 23p.

JIPSEN, Peter. ASCIIMathML.js (ver 2.0): Translating ASCII math notation to MathML and graphics. September, 2007. Disponível em < http://www1.chapman.edu/ jipsen/MathML/asciimath.html>. Acesso em 20 ago. 2010.

KAIBER, C. T.; RENZ, S. P. O Estudo de Equações Polares Utilizando o Software Maple. In: IX ENCONTRO NACIONAL DE EDUCAÇÃO MATEMÁTICA. 9., 2007. Belo Horizonte: Sociedade Brasileira de Educação Matemática.

MARIANI, Vanessa. Utilização do Software Maple no Ensino-Aprendizagem de Cálculo. 2005. Brasília: Universidade Católica de Brasília.

MINER, Robert. The Importance of MathML to Mathematics Communication. May, 2005. St. Louis: Notice of American Mathematical Society.

MINER, Robert; ION, Patrick. Mathematical Markup Language (MathML $\left.{ }^{\mathrm{TM}}\right) \mathbf{1 . 0 1}$ Specification. Revision of 7 July 1999. Disponível em <http://www.w3.org/TR/REC$M A T H M L>$. ACESSO EM 5 SET 2010.

MOODLE.ORG: OPEN-SOURCE COMMUNITY-BASED TOOLS FOR LEARNING. About Moodle. 2009. Disponível em < http://docs.moodle.org/en/About_Moodle>. Acesso em 11 out. 2009.

NOTARE, M. R.; BEHAR, P. A. O Editor Científico ROODA Exata. 2009. In: XXVII Congresso da SBC - WIE 2009 WORKSHOP SOBRE INFORMÁTICA NA ESCOLA, 2009, Bento Gonçalves. São Paulo : SBC, 2009.

PALIS, Gilda de La Rocque. Investigando alguns Desafios da Incorporação do Software Maple em Cursos Regulares do Ciclo Superior Inicial: Pré-Requisitos Algébricos e Avaliação. In: IX ENCONTRO NACIONAL DE EDUCAÇÃO MATEMÁTICA, 2007. Belo Horizonte: Sociedade Brasileira de Educação Matemática.

SILVA, Maurício Samy. Ajax com Jquery: Requisições AJAX com a simplicidade de JQuery. São Paulo: Novatec, 2009.

SMITH, Glen Gordon. FERGUSON, David. Student attrition in mathematics elearning. 2005. New Zealand: Australasian Journal of Educational Technology, v21, Number 1, 2005

WORLD WIDE WEB CONSORTIUM - W3C. Standards. 2009. Disponível em <http://www.w3.org/standards/>. Acesso em 07 nov. 2010. 